\title{
Nuevas fórmulas de comunicación con los usuarios de las bibliotecas universitarias
}

\author{
Ana CAstillo DíAz \\ Universidad de Extremadura \\ acasdia@alcazaba.unex.es \\ José Luis Herrera Morillas \\ Universidad de Extremadura \\ jlhermor@alcazabar.unex.es
}

\section{Resumen:}

Este trabajo presenta un análisis de la comunicación realizada por parte de las bibliotecas universitarias españolas a través de las nuevas las herramientas de la web 2.0 (redes sociales, plataformas de fotografías y vídeos, blogs, etc.). Estas herramientas facilitan en gran medida la interacción con los usuarios y prueba de ello es que la mayoría de las bibliotecas universitarias disponen de ellas. Debido precisamente a su alto grado de utilización y a su importante nivel de utilidad es recomendable realizar un análisis con el objetivo de establecer una serie de pautas para optimizar la comunicación que se lleva a cabo en este tipo de soportes.

Palabras clave: bibliotecas universitarias; bibliotecas universitarias 2.0; comunicación universitaria; comunicación universitaria 2.0.

\section{New ways of communication with universities libraries users'}

\begin{abstract}
:
This work presents an analysis of the use of the Web 2.0 tools by Spanish university libraries (social networks, photographs and video platforms, blogs, ...). These tools make easier the interaction with the libraries users and are implemented by the vast majority of universities. Due to its high level of use and its utility is advisable to carry out an analysis in order to establish a number of patterns useful to optimize the communication in these sites.
\end{abstract}

Key words: university libraries; university libraries 2.0 ; university communication; university communication 2.0

\section{Referencia normalizada:}

Castillo Diaz, A. y Herrera Morillas, J.L. (2014): Nuevas fórmulas de comunicación con los usuarios de las bibliotecas universitarias. Historia y Comunicación Social. Vol. 19. Núm. Especial Enero. Págs. 813-820.

Sumario: 1.Introducción. 2. Metodología 3.Resultados. 4. Conclusiones. 5. Referencias bibliográficas. 


\section{Introducción}

A lo largo de la historia, con el incremento de la competencia, la comunicación ha ido ganando posiciones en el panorama empresarial. Al margen de la competencia, o más bien, debido a ella, lograr una buena opinión pública es algo fundamental y la comunicación es una herramienta imprescindible para este propósito.

En las universidades la comunicación adquiere un papel fundamental a la hora de alcanzar la legitimación social que se les exige por parte de los diferentes públicos. Sólo es posible lograr una diferenciación positiva prestando un servicio de calidad y estableciendo una adecuada estrategia de comunicación que permita a sus públicos estar informados de las excelencias de cada marca universitaria.

En esta línea Moogan et al (2001) estiman que las entidades universitarias con marcas sólidas e información accesible y comprensible tendrán mayores oportunidades de conseguir recursos humanos y estudiantes.

Una parte importante de la comunicación universitaria hoy en día se establece a través de la World Wide Web. Así, todas las universidades españolas, sin excepción, cuentan con una sede web desde la que ofrecen información a sus stakeholders. La mayoría de estos portales suelen incluir vínculos hacia las páginas webs de las bibliotecas universitarias. Las páginas webs de las bibliotecas universitarias constituyen una herramienta de gran interés para la difusión y la gestión de los servicios bibliotecarios en las universidades.

Hace ya algunos años que las bibliotecas universitarias comenzaron a plantearse su presencia en los sitios web con el objetivo de llegar donde se encuentran sus usuarios, utilizar las mismas plataformas y canales de comunicación que ellos y seguir siendo relevantes en el contexto de su experiencia del uso de Internet (Margaix-Arnal, 2008).

Una manera de dinamizar la comunicación entre la biblioteca y los usuarios es la incorporación de herramientas de la web 2.0 como son las redes sociales, los blogs o las plataformas de fotografías y vídeos, entre otras.

En esta línea es frecuente encontrar el término 2.0 aplicado también a las bibliotecas. Así, autores como Chad (2005) o Maness (2006) hablan de las bibliotecas 2.0. Para este último autor (Maness, 2006) la biblioteca 2.0 consiste en la aplicación de tecnologías web interactivas, colaborativas y multimedia a colecciones y servicios bibliotecarios basados en la web.

Gran parte de la literatura sobre la materia coincide en señalar las siguientes herramientas como propias de la web 2.0.

- Blogs y microblogs: Los blogs nacieron como diarios personales y hace unos años los microblogs comenzaron a triunfar gracias a su agilidad comunicativa. Se trata de plataformas que permiten a los usuarios enviar y recibir mensajes de texto con una longitud máxima de 140 caracteres. Twitter hoy en día es el líder indiscutible del microblogging o nanobloging (Celaya, 2008). 
- Marcadores sociales: Servicio, normalmente gratuito, para guardar, organizar y compartir información.

- Mensajería instantánea: Se trata de una manera de comunicación, basada en el texto, que se establece en tiempo real entre dos o más personas.

- Plataformas para compartir imágenes: Sitios webs en los que los usuarios pueden almacenar, catalogar y compartir fotografías.

- Plataformas para compartir vídeos: Sitios webs en los que los usuarios pueden almacenar, catalogar y compartir vídeos.

- Podcasts: Son archivos multimedia (usualmente de audio) que emplean un sistema que permita suscribirse (RSS) y usar un programa que lo descarga para que el usuario lo reproduzca en el momento que desee (Georghegan y Klass, 2005).

- Redes sociales: Son lugares en Internet donde la gente publica y comparte todo tipo de información con terceras personas.

- RSS: Se trata de una sencilla fórmula para que los usuarios puedan recibir información actualizada sobre sus páginas web favoritas, sin necesidad de visitarlas una a una (a través de la suscripción o sindicación de contenidos).

- Wikis: Son webs que se pueden editar de manera colectiva por varios usuarios (Celaya y Herrera, 2007).

Aunque se trata de un campo cuya investigación aún se encuentra en un estado incipiente, es posible encontrar ya algunos trabajos que analizan la aplicación de los recursos 2.0 a las bibliotecas. En la tabla 1 se relacionan los principales estudios centrados en la aplicación de herramientas 2.0 a las bibliotecas:

Tabla 1. Estudios sobre la aplicación de recursos 2.0 a las bibliotecas

\begin{tabular}{|l|l|}
\hline Mensajería instantánea & Foley (2002) \\
\hline Wikis & Boeninger (2006), Fitcher (2006) \\
\hline RSS & Harinarayana (2007) \\
\hline Podcasts & Jowitt (2007),Abram, (2006) \\
\hline $\begin{array}{l}\text { Análisis de la utilización de las herramientas } \\
2.0 \text { por parte de las bibliotecas de las 100 } \\
\text { principales universidades del mundo (Times } \\
\text { Higher Education) }\end{array}$ & \\
\hline $\begin{array}{l}\text { Análisis del orden de popularidad de uso de los } \\
\text { recursos 2.0 implementados por las bibliotecas }\end{array}$ & Chua y Goh (2010) \\
\hline
\end{tabular}

Fuente: elaboración propia.

Los autores del presente trabajo realizaron un análisis previo de las páginas web de las bibliotecas universitarias españolas (Herrera, J.L.; Castillo, A., 2011). No obstante, la rápida evolución de las herramientas exige un análisis periódico con el objetivo de identificar nuevas plataformas, así como los errores y aciertos de la comunicación bibliotecaria 2.0. Por otra parte, este análisis sirve para establecer una serie de pautas a tener en cuenta en la comunicación que se lleva a cabo en este tipo de soportes. 
Este estudio persigue dos objetivos básicos. Por una parte, comprobar cuál es el proceso de asentamiento de las herramientas empleadas por parte de las bibliotecas universitarias españolas y, por otra, identificar qué otras herramientas se han podido incorporar con el paso del tiempo.

\section{Metodología}

Dos estudios previos, centrados en el análisis de las bibliotecas universitarias australianas (Linch, 2008) y chinas (Han y Quan Liu, 2009), han sido tomados como base a la hora de establecer la metodología de estudio debido a la proximidad existente con el objeto de análisis actual. En ambos casos se parte de una categorización de los recursos 2.0 a los que se les aplica una lista de evaluación, dividiendo cada categoría entre finalidad y rasgos.

La lista de evaluación ha sido adaptada a la realidad de las bibliotecas universitarias españolas. Para ello se llevó a cabo una visualización previa del conjunto de los sitios web y posteriormente se procedió a definir las categorías y los elementos de la lista de evaluación (Herrera, J.L.; Castillo; A.; 2011:183-194).

Las categorías establecidas para el análisis son las que se detallan a continuación:

- Blog.

- Compartir archivos multimedia.

- Mensajería instantánea.

- Opac 2.0. Esta variable incluye, en el trabajo de Han y Quan Liu (2009), las herramientas de navegación web, la nube de etiquetas y las alertas textuales

- Redes sociales. En este caso los elementos de evaluación se aplican a la red Facebook, por ser la red de carácter generalista más empleada por las universidades españolas (Margaix-Arnal, 2008). Además, se indica si las bibliotecas participan en otras redes de uso más reducido.

- RSS

- Otros. Esta categoría es una variable abierta que contempla otro tipo de recursos de la web 2.0 presentes aunque de manera menos significativa.

Como se indicaba en los estudios precedentes en el análisis de cada una de estas categorías se han tenido en cuenta la finalidad y los rasgos observados (Herrera; J.L., Castillo; A.; 2011: 183-194).

En total se han evaluado 64 elementos que representan el 100\% de las bibliotecas universitarias españolas que emplean herramientas de la web 2.0. El listado de las bibliotecas universitarias españolas se ha obtenido del directorio Bibliotecas universitarias y de investigación españolas: <http://bibliotecas.csic.es/otroscat/webuni. html $>$ elaborado por el CSIC (Consejo Superior de Investigaciones Científicas). 
El análisis se ha llevado a cabo en la segunda quincena de julio y la primera de agosto de 2013. Un intervalo de tiempo en el que no se suelen realizar modificaciones a las páginas webs por coincidir con la etapa vacacional.

\section{Resultados}

En términos generales se percibe cierta evolución con respecto a las versiones que podían consultarse dos años atrás en las páginas webs de las bibliotecas universitarias, sin embargo se trata de una evolución bastante leve puesto que sólo poco más del 30\% de las bibliotecas han establecido alguna modificación en sus sedes online.

La tendencia apunta al mantenimiento de las herramientas. De hecho sólo dos universidades han eliminado algún servicio que incluían en sus sedes webs: la mensajería instantánea y los blogs. Con el paso del tiempo y la aparición de otro tipo de plataformas que ofrecen una mayor interactividad e inmediatez, el uso de estas herramientas se está viendo algo más reducido. Esa es la tendencia también observada en las bibliotecas españolas.

En el otro extremo, el OPAC es el servicio que más ha visto incrementar su uso. Más del 17\% las bibliotecas, que antes no ofrecían este servicio, ahora permiten consultar sus catálogos online.

Por otra parte, sólo un $6,25 \%$ de bibliotecas ofrecen como novedad el servicio de sindicación de noticas en 2013. No obstante hay que señalar que se trata de un elemento que tiene cierta presencia en las webs de las bibliotecas universitarias españolas desde 2011.

En cuanto a las redes sociales la plataforma Facebook es la que cuenta con mayor número de adhesiones por parte de las bibliotecas españolas. Más del $9 \%$ de las bibliotecas se han decidido a abrir sus perfiles en esta red social en los últimos dos años.

En lo que respecta al empleo de otras plataformas destaca el microblog Twitter, que sigue de cerca los pasos que las bibliotecas dan en la red social Facebook, aunque con las particularidades propias del tipo de herramienta.

\section{Conclusiones}

Como se puede interpretar a través de los resultados, las bibliotecas universitarias españolas apuestan por ofrecer un servicio de OPAC online. Estas herramientas se conciben como recursos para añadir información al OPAC tradicional y suelen ofrecer opciones para que los usuarios busquen en la red o para que creen sus propias listas. 
En función del análisis realizado puede afirmarse que los blogs no se conciben tanto como una herramienta para facilitar la participación de los usuarios (para lo que se usan en mayor medida los microblogs o las redes sociales) sino más bien como apoyo a los servicios bibliotecarios.

Los chats suelen articularse a través del texto y se emplean para la atención al usuario (a pesar que de algunos permanecen inactivos). Esta herramienta vive una situación similar a los blogs, cuyo uso está viéndose limitado en favor de otras herramientas como son las redes sociales.

La sindicación de noticias suele ofrecer noticias relacionadas con eventos de las bibliotecas o, en menor medida, con novedades del catálogo.

Como se viene indicando, las redes sociales, y en concreto Facebook, es la herramienta que mayor impulso ha experimentado en los últimos años. En estos espacios, más allá del texto, las fotografías son los elementos que más se utilizan, siendo el uso de vídeos bastante más limitado. A pesar de esto hay que indicar que en los últimos años el uso de la plataforma para compartir vídeos Youtube se ha visto notablemente incrementado.

Al margen de Facebook la red social más empleada por parte de las bibliotecas universitarias españolas es Twitter. A pesar de que inicialmente se concibió como un microblog, su uso es bastante similar al de otras redes sociales.

Para finalizar, a los recursos considerados en el análisis de 2011 se debe sumar el empleo de Pinterest, una plataforma para compartir fotografías, que se añade al uso de Flickr o de Picassa con una popularidad creciente.

\section{Bibliografía}

ABRAM, Stephen (2006). Podcasts and libraries. Disponible en: http://stephenslighthouse.sirsidynix.com/archives/2006/11/podcasts_and_li.html. [06-08-2011]

BOENINGER, Chad (2006). "Using a wiki as a research guide: a year's experience", en Library Voice, july. Disponible en: http://libraryvoice.com/wikis/using-a-wikias-a-research-guide-a-years-experience. [03-09-2011].

CELAYA, J. (2008). La empresa en la web 2.0. El impacto de las redes sociales y las nuevas formas de comunicación online en la estrategia empresarial. Barcelona: Gestión 2000.

CELAYA, J.; HERRERA, P. (2007). Los blogs en la comunicación empresarial en España. Madrid: BMPO.

CHAD, Ken; MILLER, Paul (2005). Do libraries matter? The rise of library 2.0. Disponible en: http://www.talis.com/applications/downloads/white_papers/DoLibrariesMatter.pdf. [10-09-2012].

CHUA, A. Y. K. y GOH, D. H. (2010). "A study of Web 2.0 applications in library websites”. En: Library \& Information Science Research, número 32, pp. 203-211. 
DE SALAS, M.I. (2002). La comunicación empresarial a través de Internet. Valencia: Fundación Universitaria San Pablo-CEU.

FICHTER, D (2006). "Using wikis to support online collaboration in libraries". En: Information Outlook, número10 (1), pp. 30-1.

FOLEY, M. (2002). "Instant messaging reference in an academic library: a case study". En: College and Research Libraries, número 63, pp. 36-45.

GEOGHEGAN, M. W.; KLASS, D. (2005). Podcast Solutions: The Complete Guide to Podcasting. Berkeley: CA: Friends of ED.

HABIB, Michael C. (2006). Toward Academic Library 2.0: Development and Application of a Library 2.0 Methodology. A Master's Paper for the M.S. in L.S degree. Disponible en: http://etd.ils.unc.edu/dspace/handle/1901/356. [04-08-2011].

HAN, Z. y QUAN LIU, Y. (2010). "Web 2.0 applications in top Chinese university libraries". En Library Hi Tech, número 28 (1), pp. 41-62.

HARINARAYANA, N.S. y VASANTHA RAJU, N. (2010). "Web 2.0 features in university library web sites". En: The Electronic Library, número 28, (1), pp.6988.

HARINARAYANA, N.S.; KUMBAR, M. y CHAIRMAN, P. (2007). "RSS-based information services in libraries: a study". En: Library Herald, número 45, (2), pp. 130-9.

HERRERA, J.L. y CASTILLO, A. (2011). "Bibliotecas universitarias 2.0. El caso de España”. En: Investigación bibliotecológica, número 25 (55), pp. 175-200.

JOWITT, Angela (2007). "Perceptions and usage of library instructional podcasts by staff and students at Universal College of Learning (UCOL)", Support Services Dissertations and Theses, Paper 1. Disponible en: http://www.coda.ac.nz/ucol_ss_ di/1. [04-08-2011].

LINH, N. C. (2008). "A survey of the application of Web 2.0 in Australasian university libraries". En: Library Hi Tech, número 26, (4), pp. 630-53.

MARGAIX-ARNAL, D. (2008). "Las bibliotecas universitarias y Facebook: cómo y por qué estar presentes". En: El profesional de la información, número 17, (6), noviembre-diciembre, pp. 589-601.

MOOGAN, Y.; BARON, S. y BAINBRIDGE, Steve (2001). "Timings and trade-offs in the marketing of higher education courses: a conjoint approach". En: Marketing Intelligence \& Planning, número 19, (3), 179-187.

O'REILLY, T. (2007). "What is Web 2.0: Design Patterns and Business Models for the Next Generation of Software". En: Communications \& Strategies, número 65 (1), 17-37.

SUBIRATS, J. (2001). "Universidad en España: ¿época de cambios o cambio de época?”. En: Educar, número 28, pp.11-39. 


\section{Los autores}

Ana Castillo Díaz Doctora en Comunicación por la Universidad de Extremadura, donde trabaja como profesora contratada doctora en el área de Comunicación Audiovisual y Publicidad. Miembro del grupo de investigación AR-CO (área de comunicación), de la Universidad de Extremadura y de la red internacional de grupos de investigación en Comunicación y Desarrollo (Real- Code). Ha publicado diversos libros y capítulos relacionados con la comunicación empresarial e institucional. Entre ellos se encuentran: "Comunicación integral y responsabilidad social en las organizaciones", Pearson (2009); "Media and body cult", Pearson (2010). Es autora de numerosas comunicaciones presentadas en congresos nacionales e internacionales como IAMCR, ECREA, ICORIA, o los congresos de la Asociación Española de Investigación de la Comunicación (AE-IC). Ha publicado artículos en revistas nacionales e internacionales: Journal of Marketing for Higher Education, Global Journal Media, Observatorio Obs* Journal, El Profesional de la Información, Estudios del Mensaje Periodístico, Revista Latina de Comunicación Social, etc.

José Luis Herrera Morillas Doctor en Documentación por la Universidad de Granada, donde cursó también la Licenciatura en Historia del Arte y la Diplomatura en Biblioteconomía y Documentación. En la actualidad es Secretario del Departamento de Información y Documentación y Profesor Titular en la Facultad de Ciencias de la Documentación y la Comunicación de la Universidad de Extremadura, en la que imparte diferentes materias como Organización de colecciones; Información y Sociedad; o Fondo antiguo, en el Grado de Información y Documentación; y en el de Comunicación Audiovisual. También es profesor del Máster en Gestión de la Información Digital en el que imparte la asignatura Recursos de Gestión Empresarial Online. Ha publicado varios libros y artículos especializados sobre estos temas. Pertenece al grupo de investigación QUINARI de la Universidad de Exrtremadura dentro del cual participa en las líneas de investigación: Nuevas tendencias en Organización y Administración de Unidades de Información; Marketing social. Identidad e imagen corporativa en unidades de información; y Estudio y difusión de colecciones de fondo antiguo.

\section{Anexo}

Proyecto financiado por Gobierno de Extremadura y Fondos Feder.

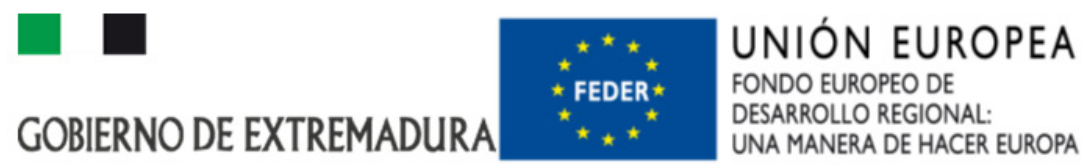

